\title{
Mujeres y minería. Resiliencias y marginaciones en territorios mineros
}

\section{Women and mining. Resilience and marginalization in mining territories}

\author{
Daniela, Escalona Thomas ${ }^{1}$ (D)
}

\begin{abstract}
RESUMEN
La irrupción territorial de la minería se legitima a través de discursos sobre desarrollo, crecimiento económico y empleo, sin embargo, en las últimas décadas se ha evidenciado que los resultados en el desarrollo local, no son lo esperados. Las mujeres no solo ven deteriorada su calidad de vida y la de sus familias producto del metabolismo espacial minero, que produce territorios al servicio de esta industria, con una identidad marcadamente masculina, sino que enfrentan dificultades para formar parte de este territorio. A través de entrevistas a actores clave de los gobiernos locales del territorio minero producido por Minera Los Pelambres en el Choapa alto, se identifica y describe tanto las limitantes que enfrentan las mujeres, así como las consecuencias en el espacio laboral, social y doméstico que sufren cuando logran -con dificultad- formar parte de la minera o insertarse en los empleos derivados de la renta minera. Se reconoce los esfuerzos de la industria por incorporar a las mujeres y con ello impactar positivamente en el territorio, sin embargo, los resultados no cumplen las expectativas de sus habitantes puesto que su transformación ha reforzado roles e identidades de género tradicionales, produciendo nuevos desafíos y limitantes para ellas.
\end{abstract}

Palabras clave: Mujeres, territorios mineros, inserción laboral, desarrollo local

\begin{abstract}
The territorial irruption of mining is legitimized through discourses about development, economic growth and employment, however, in recent decades it has been shown that the results in local development are not what was expected. For women not only see their quality of life deteriorated and that of their families as a result of the mining spatial metabolism that produces territories at the service of this industry with a markedly masculine identity, but also they face difficulties to be part of this territory. Through interviews with key informants in the mining territory produced by Minera Los Pelambres in Choapa Alto, the limitations faced by women are identified and described, as well as the consequences in the work, social and domestic space suffered when they achieve with difficulty be part of the mining company or join the jobs derived from mining rent. The industry's efforts to incorporate women and positively impact the territory are recognized, however, the results do not meet the expectations of its inhabitants since its transformation has reinforced traditional gender roles and identities, producing new challenges and limitations for them.
\end{abstract}

Keywords: Women, mining territories, labor insertion, local development. 


\section{RESUMO}

A irrupção territorial da mineração é legitimada por meio de discursos sobre desenvolvimento, crescimento econômico e emprego, porém, nas últimas décadas tem se mostrado que os resultados no desenvolvimento local não são os esperados. As mulheres não só veem deteriorada sua qualidade de vida e de suas famílias em função do metabolismo mineiro espacial que produz territórios a serviço da mineração com uma identidade marcadamente masculina, mas também elas enfrentam dificuldades para fazer parte deste território. Através de entrevistas com informantes-chave do território mineiro, produzido/ construído pela Minera Los Pelambres em Choapa Alto, são identificadas e descritas as limitações enfrentadas pelas mulheres, bem como as consequências no trabalho, no espaço social e doméstico que sofrem quando alcançam com dificuldade fazer parte da mineradora ou se inserir em empregos derivados da renda mineira. São reconhecidos os esforços da indústria para incorporar as mulheres e impactar positivamente no território; contudo, os resultados não atendem às expectativas de seus habitantes, uma vez que sua transformação reforçou os papéis e identidades tradicionais de gênero, produzindo novos desafios e limitações para eles.

Palavras-chave: Mulheres, territórios mineiros, inserção laboral, desenvolvimento local.

Chile es considerado un país minero, este sector ocupa un espacio creciente tanto en la economía nacional como en el territorio. Las inversiones en minería crecen y con ello se amplían los proyectos e impactos, transformando territorios diversos en territorios mineros (Escalona, 2020). El surgimiento de conflictos ambientales ha relevado entre otros aspectos, el rol de las mujeres como lideres de sus comunidades (Lamalice y Klein, 2016; Zambra y Arriagada, 2019), muchas veces protagonizando las resistencias. Este fenómeno hace surgir la pregunta: ¿cómo las otras mujeres, aquellas que no protagonizan o participan de las luchas, experimentan estos territorios? Estas mujeres más invisibles o invisibilizadas, ¿son resilientes a las transformaciones o bien desafían sus propias tensiones?

Las mujeres, y su rol específico en el sector y en estos territorios, han estado prácticamente ausentes. Esto no es exclusivo de Chile, variados estudios dan cuenta de la invisibilización que se hace del rol de las mujeres en minería (Lahiri-Dutt y Macintyre, 2006; Salinas y Romani, 2014; Jenkins, 2014; Caro et al.,2019) así como de los impactos multi-escalares diferenciados que sufren en estos territorios (Valdés et al.,2014; Salazar y Ramírez, 2015; Ulloa, 2016; Salazar, 2017, Macdonald, 2017), en estos trabajos se estipula que la alusión a las mujeres en el ámbito minero aparece más bien como comentarios breves dentro de otros temas importantes, más que como un debate necesario de analizar.

Un grupo dominante de aquellas investigaciones, profundizan en la inserción laboral de ellas en este sector (Consejo de Competencias Mineras, 2019 y 2020a; Consejo Minero 2019 y 2020; Caro et al., 2019). Esto es relevante, no solo porque demuestra el impacto económico local, sino porque es también una evidencia del anclaje territorial minero y de cómo, las transformaciones adquieren legitimación. Sin embargo, otras áreas son mucho menos estudiadas, como; género y medio ambiente (Salazar y Rodríguez, 2015; Salazar, 2017), los efectos sobre la familia (Valdés et al., 2014), la contribución que hacen las mujeres como mineras (Jenking, 2014; Lahiri-Dutt y Macintyre, 2006) o sobre las dinámicas de género que se producen en los territorios mineros y que afectan especialmente a las mujeres (Salinas y Barrientos, 2011; Salinas, Barrientos y Rojas, 2012; Jenkins, 2014; Ulloa, 2016; Salazar, 2017; Macdonald, 2017; Blanco y Dongo, 2019). 
Las transformaciones territoriales ocurridas en el norte de Chile, un lugar de reconocida identidad minera, tienen impactos sobre la vida de las poblaciones locales. Esta investigación se propone explorar aquellas consecuencias desde la perspectiva de género en el Valle del Choapa, donde opera minera Los Pelambres, uno de los primeros proyectos de gran minería surgidos en la oleada de inversión privada en Chile (Moguillansky, 1998).

Los avances en la caracterización de los territorios mineros necesitan ser complementados con las diferentes experiencias que allí tienen y viven las mujeres (Escalona, 2020). Lo anterior, especialmente, considerando que estos territorios producen espacios de socialización marcados por la violencia de género (Ulloa, 2016; Caro et al.,2020), en los que las mujeres son cosificadas y convertidas en bienes de consumo, y que dado su modelo territorial intensivo y extensivo que traslada trabajadores de otros territorios y países (Aroca y Atienza, 2008), genera que sus efectos se extiendan hacia espacios sociales y domésticos muy alejados a las faenas.

Por ello, con esta investigación, se pretende profundizar en uno de los múltiples aspectos que evidencia que, los efectos locales de la minería no son los proyectados en términos de desarrollo y bienestar, por el contrario, muchas veces perjudican las trayectorias territoriales propias sacrificando social y ambientalmente a sus habitantes por cuestionables beneficios económicos. Este aspecto, se refiere a las relaciones diversas que establecen las mujeres en territorios mineros, cuya experiencia muestra que las dinámicas de género producidas por este modelo territorial perpetúan y profundizan desigualdades que ellas deben asumir, resistir o ambas.

La sistematización de estas dinámicas de género en territorios mineros permitió visibilizar en primer término, la importancia que ha adquirido en las últimas décadas las limitadas posibilidades de inserción de las mujeres en la gran minería, esto abarca las diferencias en cuanto a ocupaciones y rangos, así como, las dificultades que impone el trabajo por turno, característico de esta industria. Segundo, las limitaciones que enfrentan las mujeres que deciden formar parte del desarrollo minero, tanto en su inserción directa en la industria, así como las mujeres que participan de actividades derivadas de la renta minera, principalmente servicios. Esta relación es resiliente y contradictoria, ya que, por una parte, critican la industria identificando las tensiones que para ellas tienen los espacios laborales masculinizados, con una marcada construcción del género que las limita a ciertas labores y tareas en jerarquías y condiciones inferiores, pero, por otra parte, necesitan emplearse, y en sus localidades los empleos están restringidos a la industria. Esta marginación tiene consecuencias en el espacio social y doméstico, puesto que se tensiona la construcción de género tradicional de la minería y las posibilidades que ellas ven en este nuevo rol lleno de proyecciones y promesas.

\section{El Valle del Choapa y su transformación en territorio minero}

El Valle del Choapa está localizado en el norte de Chile y se enmarca en el área de los valles transversales, específicamente es el valle meridional de esta macrozona formado por el rio del mismo nombre. La intervención del proyecto minero recorre la totalidad del valle, 120 kilómetros de cordillera a mar, en un entorno donde coexisten más de 40 localidades con 84.000 habitantes (Censo, 2017). Sus pueblos desarrollan actividades económicas tradicionales, tales como la 
agricultura, ganadería de subsistencia, pesca y minería pequeña y mediana (Escalona, 2020). Comprende a las comunas de Salamanca, Illapel, Canela y Los Vilos, sin embargo, solo las dos primeras se han visto reconfiguradas como territorios mineros, puesto que Los Vilos y Canela, con menos impacto económico -pero no ambiental- continúan teniendo otras actividades primarias como fuente de ingresos.

El yacimiento a rajo abierto de Minera Los Pelambres, se sitúa en la parte alta de la Cordillera de los Andes. Comienza sus operaciones en 1992, extrayendo principalmente cobre. La intervención además del rajo de mina y la planta de procesos incluye, 2 tranques de relaves, minero-ductos y relave-ductos que afectan a la totalidad del valle agrícola. El proyecto pertenece a la compañía de capitales nacionales Antofagasta Minerals, que se encuentra entre los diez mayores productores de cobre del mundo. La planta concentradora, ubicada a $1.600 \mathrm{msnm}$, procesa 210.000 kilo/ toneladas de mineral a diario. El funcionamiento de la mina ha sido proyectado con una vida útil de 30 años (SEIA, 1997).

Las comunas que se consideran como territorios mineros han sido reconfiguradas por este proyecto en su fase expansiva, cuando pasa desde Company town (Garcés, 2003) a región comoditi (Daher, 2003), estas transformaciones son resultado de los esfuerzos del Estado para organizar los territorios en relación a la disponibilidad de recursos naturales (Bustos-Gallardo y Prieto, 2019), estos autores indican que esta transformación consiste en primero, la producción de paisajes, luego la transformación metabólica del ecosistema, seguida de procesos discursivos y materiales que construyen nuevas identidades y originan nuevos conflictos. Cada una de estas transformaciones ha tenido un impacto significativo y diferenciado en las mujeres.

Es en las áreas urbanas donde se concentran las mayores transformaciones. Salamanca, por ejemplo, es la comuna que alberga las principales intervenciones de Minera Los Pelambres (MPL), la que en términos demográficos presenta un índice de masculinidad de 115,23 hombres por cada 100 mujeres, mayor a la media nacional de 95,9 (Censo,2017), presentando una alta población flotante de migrantes internos y externos, actualmente es la región de Coquimbo la que presenta el mayor atractivo migratorio del país (Censo, 2017). Por otra parte, Illapel, comuna con una larga tradición de mediana y pequeña minería, cuenta con un índice masculinidad del 94,8 qué, aunque se encuentra más cercano a la media nacional, al mismo tiempo cuenta con una actividad económica que se ha concentrado en los servicios a la minería, entre las que se encuentran las empresas subcontratistas. En ambas comunas es posible observar unas cuantas cuadras del centro comercial ocupadas por locales nocturnos.

Estas transformaciones territoriales, tienen efectos en la vida social y doméstica, específicamente en las mujeres que los habitan, ellas experimentan las tensiones producto de un territorio al servicio del desarrollo minero, en el cual deben asumir los roles que estas dinámicas territoriales les asignan.

\section{La cultura minera local desde la perspectiva de género}

La perspectiva de género permite visibilizar, comprender y abordar los problemas y las desigualdades que enfrentan las mujeres, especialmente las mujeres pobres en territorios marginales 
(Lagarde, 1996). A su vez el género, como categoría de análisis, permite entrever las relaciones que se establecen en estos territorios para desde allí, identificar los factores que reproducen las inequidades en las distintas escalas, así como los efectos que esto tiene en la vida de las mujeres de las comunas de Salamanca e Illapel.

La cosmovisión de género cambia con las culturas y las sociedades (Lagarde, 1996), por tanto, los territorios mineros construyen su propia visión de género. Las mujeres también forman parte de esta cultura minera masculinizada, pero para ellas se produce tensión. Este trabajo pretende identificar las múltiples relaciones que construyen las mujeres en estos territorios, específicamente las mujeres que experimentan este territorio y cuyas marginaciones son más invisibles.

Para ello, en primer lugar, se realizó una revisión bibliográfica para abordar la visión y evolución que la industria ha tenido y tiene respecto a la inclusión de las mujeres en el trabajo minero. En segundo lugar, se realizaron entrevistas a informantes clave de los gobiernos locales de las comunas de Salamanca e Illapel: las encargadas de los Programas de Jefas de Hogar, las/los encargados de la Casa de la Mujer, los encargados de las Patentes Municipales y las encargadas de las Oficinas Municipales de Inserción Laboral (en adelante OMIL) de las comunas de Salamanca e Illapel, más una última entrevista a la profesional a cargo del Policlínico de Salamanca (Policlínico de Transmisión Sexual-PTS), en total 9 entrevistas en profundidad. Además, se realizaron entrevistas informales, en los servicios regionales: Ministerio de Minería, Sernam y Sernageomin de la Región de Coquimbo, complementadas con observación y notas de campo realizadas durante la visita a terreno en agosto de 2019.

La mayor parte de las entrevistadas fueron mujeres, salvo por los encargados de las patentes municipales. Algunas de ellas habían trabajado anteriormente en Minera Los Pelambres o en empresas contratistas, por tanto, su experiencia también formó parte de la visión de las dinámicas de género.

\section{Empleo femenino en la minería masculina}

Como se mencionó anteriormente, el rol de las mujeres en la minería y en los territorios producidos por ella, es una cuestión débilmente investigada. Sin embargo, aunque existen diversos estudios que analizan la situación de desigualdad que ha afectado a las mujeres en lo económico, político, social y laboral en la industria extractiva (Gutiérrez, 2007, Salazar, 2017;Macdonald, 2017, Blanco y Dongo, 2019; De la puente, 2017; Gomez y Angelcos, 2018) son menos los que profundizan en la dimensión de género en la minería (Eftimie, et al., 2009; Salinas y Romani, 2014; Jiménez, et al., 2014; Stefanovic y Saavedra, 2016; Ulloa, 2016; Angelcos, 2017; Caro et al., 2019; Caro et al., 2020, Salazar y Rodriguez, 2015), los que en su mayoría se aproximan desde la inserción laboral de las mujeres en el sector minero. Este tópico es el que concentra la preocupación de la industria y del Estado, puesto que la participación laboral de las mujeres es un aspecto central en los debates sobre desarrollo (Macdonald, 2017). Por tanto, no solo significaría ser una sociedad más inclusiva, sino también se ha demostrado que su incorporación tiene beneficios para la productividad e innovación (Stefanovic y Saavedra, 2016; Caro, et al., 2019), pudiéndose también establecer que en los hogares donde la mujer aporta una parte mayoritaria del ingreso familiar tienden a aumentar 
el nivel educacional de los hijos y por tanto la calidad de vida en general (Salazar, 2017; Consejo de Competencias Mineras, 2020a).

Adicionalmente, el interés en la inserción laboral de las mujeres en la minería, se relaciona con las narrativas usadas por la industria minera para su legitimación, los empleos con mejores salarios en comparación con otras industrias, así como los beneficios para el desarrollo local (Bebbington, 2013; Jenkins 2014), constituyen parte del imaginario instalado, sin embargo, al profundizar en los territorios locales metabolizados por esta actividad, dichos beneficios son cuestionados (Blanco y Dongo, 2013), lo que especialmente sucede con las mujeres que afectadas en múltiples dimensiones (Eftimie et al., 2009; Jenkins, 2014; Salazar y Rodríguez, 2015; Ulloa, 2016; Macdonalds, 2017). Estos impactos diferenciados para ellas ocurren no solo desde lo laboral, sino también en lo social y doméstico, estableciendo que los territorios mineros no son amables para las mujeres y sus hijos, las que adicionalmente sufren mayores consecuencias por el deterioro ambiental (Salazar y Rodríguez, 2015).

En el aspecto laboral, los efectos son múltiples, en primer lugar, al ser la minería una industria masculinizada el número de empleos directos para las mujeres son mucho menos que los ofertados para los hombres, en este punto los limitados empleos ofrecidos para las poblaciones locales normalmente son ocupados por ellos (Jiménez et al., 2014; Gómez y Angelcos, 2018; Caro et al., 2019; Blanco y Dongo, 2019). Segundo, las jornadas laborales por turnos, son una limitante para las mujeres encargadas tradicionalmente de las tareas de cuidado y labores domésticas (Salinas y Romani, 2014; Stefanovic y Saavedra, 2016). Tercero, insertarse en una industria de hombres, implica cambios estructurales y logísticos que muchas empresas no están dispuestas a hacer (Blanco y Dongo, 2019; Caro et al., 2019). Cuarto, las asimetrías salariales que afectan a las mujeres también ocurren en la industria extractiva minera (Blanco y Dongo, 2019) y finalmente, la industria extractiva en general tiene fuertes impactos en las actividades económicas tradicionales, afectando la capacidad de las mujeres de generar recursos propios y tener una autonomía económica.

\section{Antecedentes sobre la inserción laboral femenina en la industria minera}

A nivel global, entre el $70-80 \%$ de las mujeres trabaja en servicios (OIT, 2020), esto significa que su inserción en la industria extractiva es muy baja, no superando el $10 \%$ de la fuerza laboral (Eftimie et al. 2009). En la industria minera esto se traduce en empleos de menor jerarquía y por tanto con menor salario, garantías y beneficios (Lahiri-Dutt, 2010). En América Latina, la CEPAL estima que la minería y los hidrocarburos emplean a menos del $1 \%$ de la población ocupada (Stefanovic y Saavedra, 2016; De La Puente, 2017) es decir, su impacto en la empleabilidad de la región es pequeña en relación con su participación de la economía. De los empleos ofrecidos por la industria minera en particular, solo el $12 \%$ es ocupado por mujeres, volumen similar a lo que ocurre a nivel global, con lo cual la presencia masculina es abrumadora (Stefanovic y Saavedra, 2016).

Con respecto a los territorios donde se inserta la industria extractiva minera, la mayor parte de los proyectos mineros se ubican en lugares alejados o empobrecidos (Svampa y Antonelli, 2009; Lamalice y Klein, 2016) y en estos territorios pobres con un alto porcentaje de población rural, las mujeres tienden a estar insuficientemente preparadas para solicitar puestos de trabajo en las 
empresas mineras, ya que generalmente tienen bajos niveles de educación y pocas calificaciones (Ward y Strongman 2011).

En Chile, aunque la participación laboral de las mujeres aumenta en las últimas décadas, sigue siendo la más baja de la región, con brechas importantes de más de 10 puntos porcentuales respecto de países de similar nivel de desarrollo socio económico (Contreras y Plaza, 2010). A esta escala la tasa de participación femenina es de 52,7\% y la de los hombres es de un $73,8 \%$ (INE, 2019). Esta menor participación estaría influenciada por creencias tradicionales respecto de los roles femeninos que condicionan que menos mujeres vean una opción o tengan una oportunidad en el trabajo remunerado (Contreras y Plaza, 2010), puesto que el principal motivo para no buscar empleo se debe al cuidado de la familia (INE, 2019).

Estas creencias tradicionales influyen en que el sector minero no constituya un rubro posible para las mujeres, a pesar de ser considerado uno de los principales para el desarrollo del país. Las mujeres ocupan sólo el 8,4\% de los empleos ofrecidos (Consejo de Competencias Mineras, 2019), estableciéndose diferencias entre empleos directos y subcontratados, mientras en las primeras llega a 8,9\% (2018) en las segundas la participación no supera el 5,5\% (Consejo de Competencias Mineras, 2020a). Por ejemplo, en la principal región minera del país Antofagasta, la participación laboral de las mujeres es menor que a nivel nacional y se concentra en el área de servicios 38,3\% y comercio 35,9\% (Salinas et al., 2010). La activación del sector terciario se relaciona con que los ingresos generados por la minería tienen efectos en la demanda por servicios y bienes. De hecho, la gran minería para demostrar su impacto económico territorial, multiplica los empleos directos por un factor de 2.55 para estimar los empleos indirectos generados por la renta minera (Consejo de Competencias Mineras, 2019). Estos empleos en servicios y comercio efectivamente tienen menos barreras de género, pero circunscriben a las mujeres a desempeñar roles entendidos como tradicionalmente femeninos (SERNAM, 2009; Salinas y Barrientos, 2011).

Esta construcción de género en la minería se ve reflejada en que solo en 1990 ingresó el primer grupo de mujeres a una mina en Chile, fue al proyecto El Teniente de la empresa nacional CODELCO. El grupo estuvo conformado por esposas de los trabajadores, para evitar el rechazo de los mineros (CODELCO, 2007), posteriormente, en 1996 se permite legalmente el ingreso de las mujeres a los yacimientos mineros subterráneos (Código del Trabajo, 2018). Desde este momento, su inserción ha sido lenta debido a las múltiples dificultades que se han identificado en la industria extractiva (Waziers y Morales, 2020).

\section{La construcción de género en la minería}

De acuerdo a la investigación de Barrientos (et al., 2009) histórica y culturalmente la minería, como empleo, constituye un mecanismo de realización para los hombres, ya que, a través de éste forman un hogar, son proveedores y así cumplen los roles socioculturales esperados para ellos (Olavarría, 2000). De acuerdo a Salinas y Barrientos (2011), los hombres se encuentran socialmente exigidos por una dinámica en la que trabajo-dignidad y capacidad se relacionan en un círculo vicioso y que condiciona su identidad masculina adulta (Salinas y Arancibia, 2006; Salinas, 2007). De este modo, la dominación que ejercen se manifiesta en la discriminación contra las mujeres, vinculado con el proceso de construcción de su propia identidad. Así en la minería, se recrean 
verdaderos rituales de hombría, a través de un lenguaje soez, bromas y rumores (Barrientos et al., 2009), sexualizando los discursos, demostrando experiencia y conocimiento de los significados sexuales.

Esto se observa, igualmente, en las interacciones en el espacio social (Barrientos et al., 2009), ya que para los hombres estos lugares son sitios de distracción (Salinas y Barrientos, 2011), es decir, los que ocurre en los lugares de trabajo, se refleja en el territorio minero en todas las escalas (Salinas y Barrientos, 2011; Valdés et al., 2014; Caro et al., 2019), metabolizando el paisaje general, desde uno múltiple y diverso a uno centrado en dispositivos para la minería y sus trabajadores.

Las mujeres que trabajan en estos contextos reproducen relaciones de poder asimétricas y de subordinación, lo que contribuye a reforzar identidades de género y modelos relacionales de inequidad, que son aceptados en este entorno productivo (Salinas y Barrientos, 2011, Caro et al., 2020) y que son reproducidos en el espacio social y doméstico.

Por tanto, la incorporación e inclusión de las mujeres dentro de la industria minera en general y en las faenas mineras en particular, está condicionado por una serie de elementos que se relacionan a los estereotipos tradicionales de género sobre la minería como 'trabajo de hombres' (Lahiri-Dutt y Macintyre, 2006; Eftimie et al., 2009). Éstos han contribuido a que la participación y la contribución de las mujeres en este sector haya sido invisibilizado.

Dentro de los motivos que están detrás de la débil inserción de las mujeres en el sector minero, y muchos de estos motivos también son visibles en la pequeña y mediana minería. Algunas perspectivas indican que las condiciones de exigencias físicas, los riesgos para la salud, así como las condiciones ambientales extremas la hizo una actividad considerada para hombres (Ayala, 2012). Por otra parte, existen creencias y mitos que se han construido en este sector para alejar a las mujeres -así como otros sectores masculinizados como la pesca- debido a creencias como que es mala suerte que las mujeres ingresen a las minas (especialmente cuando menstrúan) (Lahiri-Dutt 2011 en Jenkins, 2014; Ulloa, 2016).

Probablemente a partir de estas creencias, es que en Chile se establece por norma que el ingreso de las mujeres a faenas mineras subterráneas estuviera prohibido. Sin duda, esta normativa incidió en generar una cultura minera que reforzó la exclusión femenina mediante un imaginario de mitos y supersticiones en torno a este espacio de trabajo (Campos, et al., 2016; Caro, et al., 2019). La industria declara que un hito en este camino de ampliación fue cuando en 2018 , a través del acuerdo público-privado denominado "Decálogo de la Industria Minera por la Incorporación de Mujeres y la Conciliación de la Vida Laboral, Familiar y Personal", se estableció un marco de trabajo que ha orientado las acciones que la industria desarrolla para ir transformando dicha cultura minera (Consejo Minero, 2020).

A partir de este periodo, hay una tendencia por parte de las empresas a reivindicar a la minería como una alternativa atractiva de empleo para las mujeres. La industria minera, a nivel gremial a promovido el ingreso de ellas, no solo porque son consideradas buenas empleadas (Consejo Minero, 2019; Consejo de Competencias Mineras, 2020a) sino porque en las empresas se han reconocido los beneficios para la productividad y la generación de innovación que conlleva contar con equipos mixtos (Stefanovic y Saavedra, 2016). 


\section{Las mujeres del Choapa alto y su inserción en territorio minero de MLP}

En la gran minería la participación de la mano de obra local se ha reducido de un $82,1 \%$ en 2012 a 74,6\% en 2019 (Consejo de Competencias Mineras, 2020b). De acuerdo con la información publicada por Minera Los Pelambres, existe un compromiso con la provincia del Choapa para cumplir con la empleabilidad, donde el $30 \%$ de los contratos directos debe ser mano de obra local (Minera Los Pelambres, 2017), muy por debajo a la declarada por la industria a nivel nacional.

Este compromiso de la empresa con el empleo local se gestiona desde el 2015, a través de un portal de empleo que es la plataforma de comunicación entre la empresa y los municipios de la provincia. En este acuerdo, en el que no se establece una cuota de género, no existe ningún mecanismo de fiscalización por parte de los interesados, tarea adicional que deben realizar, puesto que las Oficinas Municipales de Inserción Laboral de las comunas de Salamanca e Illapel no reciben información que acredite su cumplimiento (Comunicación personal, OMIL Salamanca 13 agosto 2019 y OMIL Illapel 14 agosto 2019). Por el contrario, se indica que hay ocasiones en que "las personas que se han contratado luego son despedidas" (Comunicación personal, OMIL Salamanca 13 agosto 2019), por tanto, el cumplimiento en cuanto al número de contratos no significa un compromiso de permanencia.

Adicionalmente, para fortalecer la inserción local en el trabajo minero se desarrolla un programa de capacitación para que efectivamente estos habitantes, sin distinción de género, puedan acceder a los puestos ofrecidos. Estas capacitaciones se generan desde los distintos servicios encargados del Estado, pero también muchos de estos programas resultan de la coordinación de con los municipios (Comunicación personal, OMIL Salamanca 13 agosto 2019 y OMIL Illapel 14 agosto 2019).

La información entregada por ambas Oficinas Municipales de Inserción Laboral, indican que la mayor parte de los puestos de nivel técnico-profesional que actualmente ocupan las mujeres en MPL de estas comunas, son "contadas con los dedos de la mano" (Comunicación personal, OMIL Salamanca 13 agosto 2019), es decir, los datos publicados por la industria sobre la inserción de la mujer en nivel técnico y profesional del 27\% (Consejo de Competencias mineras, 2020a), no correspondería a la mano de obra local, sino que estos cargos son desempeñados por mujeres que son trasladadas desde otras regiones e incluso países.

La mayoría de las mujeres que se emplean en la gran minería corresponde a puestos administrativos, de relaciones públicas y de servicios (Competencias de Competencias Mineras, 2020a). Mientras que la inserción técnico-profesional local en la operación minera se ha enfocado en la prevención de riesgos. De acuerdo con las encargadas, ellas son consideradas "un mal necesario" (Comunicación personal, OMIL Salamanca 13 agosto 2019) ya que las contratan por obligatoriedad de la ley, pero son consideradas "un estorbo, nadie las quiere, si no fuera obligatorio no las tendrían" (Comunicación personal, OMIL Salamanca 13 agosto 2019). Este aspecto, refuerza la idea que la inclusión de las mujeres no solo se relaciona con el número de empleos ofrecidos, sino que actualmente el tipo de empleos y las condiciones en las que las mujeres deben ejercerlos son discriminatorias. 
Como se mencionó, la mayor parte de mujeres que logran insertarse en la industria minera son un porcentaje menor del total de trabajadores del sector $(8,4 \%)$ y esto se refleja en la situación del Valle del Choapa. Puesto que, efectivamente no se insertan en la minería directamente, mucho menos en empleos de nivel técnico o profesional, como se explica en el párrafo anterior, sino que acceden a los trabajos en el área de servicios dentro de la minera o bien en empresas contratistas de la minera. A escala local este tipo de empleos se asocia a precariedad laboral y bajos salarios. La información recabada indica que, la mayoría de las mujeres que trabajan en la zona, lo hace fuera de la mina, accediendo a trabajos a trato, de medio tiempo, con salarios que no alcanzan el salario mínimo, y que a pesar de ello son escasos y muchas veces deben competir por obtenerlos (Comunicación personal, OMIL Salamanca 13 agosto 2019; OMIL Illapel 14 agosto 2019; Programa jefas de hogar Salamanca 12 agosto 2019 y Programa jefas de hogar Illapel 14 agosto 2019).

La falta de empleos para ellas las lleva a buscar en sectores que se fortalecen con la renta minera, pero que constituyen rubros menos aceptados socialmente, como los bares, cabaret y la prostitución (Comunicación personal: Programa jefas de hogar Salamanca 12 agosto 2019; Programa jefas de hogar Illapel 14 agosto 2019 y Policlínico Transmisión Sexual 13 agosto 2019), que afectan directamente a las mujeres, debido a su vínculo con la violencia y la discriminación social (Salinas y Barrientos, 2011). Muchas mujeres optan por estos empleos, no solo porque son los más recurrentes en el lugar, sino porque muchas veces son los únicos a los que pueden optar. Esto las hace aún más vulnerables en términos sociales y económicos, aumentando la precariedad laboral $y$, además, cargando con una serie de imaginarios sociales en torno a ellas y a los lugares en los que trabajan.

En definitiva, los problemas identificados en torno a la inserción de las mujeres locales en la minería se relacionan, en primer lugar, con una falta de oferta de empleos para ellas, pero también, con características de aquellos empleos que no los hacen compatibles con sus otros roles, así como, que aseguren un trato no discriminatorio en términos de las jerarquías y de las infraestructuras necesarias para su integración, toda vez, que en estas localidades existe una dependencia de los empleos ofrecidos por la empresa.

Las relaciones que produce la industria minera con los territorios, específicamente con las mujeres, dispone una construcción de género donde ellas están marginadas de las dinámicas económicas, y con ello de las posibilidades de transformar dichas relaciones.

\section{Limitantes y desafíos de las mujeres locales para su inserción en el territorio minero}

Para que la minería logre procesos de desarrollo local con perspectiva de género, no es suficiente con emplear a un mayor número de mujeres, sorteando las creencias y mitos sobre ellas o la cultura masculina al interior de las faenas. Como se ha mencionado hay problemas estructurales que deben abordarse en toda la industria en términos de igualdad de género, en particular en relación con la cantidad y tipo de oferta de empleos, el trabajo por turnos, la igualdad salarial y la seguridad adecuada, el equipamiento, las instalaciones separadas y apropiadas (Blanco y Dongo, 2019; Caro et al., 2019), a lo que se debe agregar el problema de los empleos de menor rango, acoso sexual y violencia de género (Eftimie et al., 2009; Women in mining, 2020), aspectos que 
fueron identificados tanto por las encargadas municipales de los Programas de Jefas de hogar como por las Oficinas de empleos de ambas comunas.

De acuerdo con los antecedentes empíricos se establecieron las principales dificultades que tienen las mujeres de estas comunas para participar de los procesos económicos producidos por la minería, los que se clasificaron en tres: las dificultades que enfrentan las mujeres en su inserción formal en Minera Los Pelambres y las empresas contratistas; los desafíos que este tipo de empleos supone en el espacio doméstico y tercero, las limitantes que experimentan con la marginación en lo económico, social y doméstico.

En primer término, la inserción formal de las mujeres en la industria, está restringida tanto por el número de ingresos como también por las dificultades que deben enfrentar y que condicionan su permanencia (Román et al., 2013). Algunos tópicos que siempre están al centro de los análisis de género, corresponden a la igualdad salarial y jerarquía de los puestos de trabajo. Con respecto al primero, no aparecieron en las entrevistas realizadas antecedentes relevantes que dieran cuenta de una situación desigual para las mujeres en MLP, probablemente porque este análisis se centra en los habitantes locales y ellas no tiene una representatividad alta dentro de los trabajadores de la empresa como tampoco acceso a puestos de jerarquías elevadas en los cuales normalmente se establecen diferencias salariales más significativas (Comunicación personal, OMIL Salamanca 13 agosto 2019; OMIL Illapel 14 agosto 2019). Sin embargo, con respecto a las jerarquías de los puestos de trabajo a los que ellas pueden acceder, si se identifica una desventaja, puesto que los tipos de empleo que ofrece la minera para las mujeres locales son básicamente servicios de limpieza del hotel de mina, limpieza de oficinas y otros recintos, lavado de la ropa del campamento minero y servicios de alimentación, todos ellos a través de empresas contratistas (Comunicación personal, OMIL Salamanca 13 agosto 2019).

Con respecto a las capacitaciones a las cuales acceden las mujeres para sortear esta desventaja en el acceso a los empleos ofrecidos por la minera, tanto las capacitaciones de la empresa como las ofrecidas por el Estado no tienen distinción de género como requisito o condicionante, sin embargo, en la práctica las mujeres y los hombres optan por los temas vinculados a lo que tradicionalmente se asigna a su género. Es decir, ellas eligen capacitaciones en áreas de repostería, banquetería, cocina internacional y peluquería, y aunque también llegan cursos de electricidad u otras labores, esos cursos no logran llenar los cupos solo con mujeres (Comunicación personal Programa jefas de hogar Illapel 14 agosto 2019 y Casa de la mujer Illapel 15 agosto 2019).

Esto ocurre, no solo porque estas localidades son tradicionales con respecto a los roles de género lo que es fortalecido por la minería, sino también debido a que, cuando las mujeres optan por capacitaciones tradicionalmente para hombres como construcción o electricidad, aunque las finalicen con éxito no logran ser contratadas por las empresas de la zona, por prejuicios de género (Comunicación personal Programa jefas de hogar Illapel 14 agosto 2019).

En el caso de las empresas contratistas, las cuales no tienen compromisos formales para la contratación de población local ni tampoco de cuotas de género, los antecedentes recogidos en terreno indican que el principal obstáculo es el equipamiento y la infraestructura separada (Comunicación personal, OMIL Salamanca 13 agosto 2019) puesto que estas empresas construyeron sus instalaciones en periodos en que no existía esta demanda y muchas de ellas cuentan con un 
baño o un camarín, por lo tanto, la contratación de mujeres significa invertir en nueva infraestructura, que muchos no están dispuestos a hacer.

Segundo, el desafío de conciliar el trabajo con la familia y la vida personal (Gómez y Angelcos, 2018; Caro et al., 2019), factor que resultaría crítico al momento de decidir ingresar o permanecer en la industria minera pero que aplica para el mercado laboral en general (Gutiérrez, 2007). En este punto, resulta especialmente compleja la tipología del trabajo en faenas mineras, debido al sistema de turnos con el que se opera, a lo que se suma la lejanía de sus hogares y la escasa conectividad que caracteriza estos territorios. Este modelo de trabajo que tiene implicancias territoriales muy amplias, desafía a las mujeres, especialmente a aquellas que han formado familia y/o tienen hijos.

La tipología del trabajo por turnos al que deben someterse en caso de postular y quedar, las obliga a abandonar sus hogares y muchas veces a sus hijos, por lo que la principal condicionante es la red de apoyo familiar, normalmente la madre o la suegra, para encargar el cuidado de sus hijos o hijas. Este modelo también aplica para las mujeres locales, a pesar de que puedan estar más cerca de sus hogares, igualmente son obligadas a pernoctar los días de faena en los lugares establecidos, que pueden ser dentro de la mina o bien en los pueblos contiguos, Cuncumén o Chillepín.

Frecuentemente, de acuerdo a las entrevistadas, la red de apoyo familiar se puede llegar a transformar en una dificultad. Puesto que, después de un tiempo las cuidadoras no quieren entregar a los niños a sus madres, generando un conflicto entre madre e hija o entre suegra y nuera, por el cuidado de los niños. En casos en que no cuenten con esta red de apoyo, y sobre todo para las jefas de hogar (las que más necesitan trabajar), el salario debe permitirles pagar por el cuidado de los hijos. No obstante, debido a los trabajos a los que ellas pueden optar, con salarios que se consideran "buenos para la zona, pero bajos para la minería" (Comunicación personal, Casa de la Mujer Illapel 15 agosto 2019), no les alcanza para costear el cuidado de los hijos. Por lo tanto, los empleos que ofrece la minería para las mujeres de estas localidades no son una opción que funcione para ellas. A esto se añade también la condena social a la que se exponen, puesto que en una sociedad tradicional el alejamiento de la madre implica cuestionamientos como, "tiene a su niña botada en la casa" (Comunicación personal, Programa jefas de hogar Salamanca 12 agosto 2019) o "es el día de la madre, ¿cómo vas a trabajar?" (Comunicación personal, OMIL Salamanca 13 agosto 2019), constituyen parte de los relatos con que cargan estas mujeres.

La tercera limitante, se relaciona con que ellas deben sortear una "cultura de discriminación" (Caro et al., 2019). En ambientes altamente masculinizados como la minería (Díaz, 2014, Angelcos, 2017; Gómez y Angelcos, 2018; Caro et al., 2019), resultan habituales prácticas relacionadas con la discriminación de género que constituyen barreras simbólicas a la inserción femenina (Gómez y Angelcos, 2018). Esto se evidencia en un trato denostativo hacia la mujer, a través del hostigamiento sexual y la invisibilización de sus contribuciones y opiniones. Lo anterior deriva en la necesidad constante de las trabajadoras de tener que validarse frente a sus pares y superiores, especialmente en aquellos puestos donde su presencia es más escasa (Caro et al, 2019). Dentro de esta cultura discriminatoria en las faenas mineras, se establece que las mujeres solteras y jóvenes, "vienen a buscar marido" (Comunicación personal, OMIL Salamanca 13 agosto 2019), por lo que ellas deben diseñar estrategias para relacionarse en estos espacios, utilizando roles de hija, 
buscando protección en otras mujeres u hombres, o como madre, estableciéndose como cuidadoras de sus pares. Sumado a ello, en roles técnicos dentro de la faena, los cuestionamientos son mayores, puesto que tienen a hombres como subalternos teniendo que sortear frecuentes desconfianzas de sus conocimientos solo por el hecho de ser mujeres (Comunicación personal, OMIL Salamanca 13 agosto 2019).

En definitiva, las dificultades impuestas por el modelo de trabajo y la cultura minera, influyen en la débil incorporación de las mujeres en las faenas mineras, y explican que en mayor medida trabajen en los empleos indirectos de la minería, aquellos considerados como parte del efecto multiplicador de la renta minera; comercio, servicios generales y servicio doméstico.

\section{Otras dinámicas de género asociadas al impacto local minero}

El valle del Choapa tiene una vocación agrícola pero también minera. Por tanto, las transformaciones de la gran minería, implicaron nuevos metabolismos pero a su vez, heredaron una cultura minera local, especialmente en Illapel.

Este metabolismo, genera redes espaciales entre los territorios mineros, sus alrededores y sus impactos territoriales. Por ejemplo, a través de las migraciones temporales o permanentes, principalmente de hombres, hacia los enclaves mineros, obligándolos al distanciamiento de sus familias que permanecen en lugares lejanos, se generan procesos de espacialización, tanto, de la producción ya que deben permanecer cerca de los sitios de extracción, como de la reproducción que ocurre en sus hogares con esta temporalidad particular así como con los traslados frecuentes. Todo ello perpetúa roles de género y ahonda desigualdades económicas, sociales y políticas (Ulloa, 2016). De este modo, tanto la tradición de los territorios estudiados como el metabolismo asociado al extractivismo minero de las últimas décadas, impone dinámicas de género que reproducen desigualdad y fortalecen roles tradicionales.

La minería refuerza estas dinámicas, a través del proceso mencionado, pero también debido a que la gran minería ha instalado un modelo de fidelización de la fuerza de trabajo a través de los beneficios otorgados a las familias de los trabajadores, consagrando así lo que Valdés (et al., 2014) ha denominado familia industrial, donde el hombre es el proveedor gracias a los altos salarios de la minería y la mujer la responsable del hogar. Situación diferente ocurre con los trabajadores subcontratados que no gozan de los mismos beneficios pero que culturalmente siguen este patrón.

De esta forma la minería sigue asociada a lo masculino, no solo por las diferencias físicas entre hombres y mujeres o por las razones enunciadas en el plano de los mitos como se mencionó anteriormente, sino también porque ha configurado un modelo territorial que continúa reproduciendo las prácticas que mantienen las desigualdades de género.

Por otra parte, los territorios estudiados, distantes, con alta población rural y niveles de pobreza por encima de la media nacional, determinan y condicionan las identidades territoriales, 
refiriendo que las mujeres locales, "son tradicionales y conservadoras por tanto les cuesta tomar la decisión de trabajar y aún más de trabajar en la mina" (Comunicación personal Programa jefas de hogar Illapel 14 agosto 2019). Por tanto, es fundamental que un proyecto minero, produzca desarrollo en las localidades donde se instala, incluyendo las complejidades de estas comunidades y sus habitantes.

Los efectos no deseados que provoca la minería en los territorios locales, no solo representan limitantes en los procesos de desarrollo, sino también producen nuevas tensiones y problemáticas. Uno de los antecedentes que se recabaron y que sin duda constituye un ámbito en que es necesario profundizar, es la relación entre minería y prostitución. La comuna de Salamanca, es la comuna de la Región de Coquimbo, que tiene el mayor número de trabajadoras sexuales controladas de acuerdo a los antecedentes del Hospital comunal, incluso considerando que desde 2007 el control sanitario ya no es obligatorio (Decreto No 206 del Ministerio de Salud). El vínculo entre esta actividad y la minería se reconoce en las comunidades estudiadas como histórico y cultural. Sin embargo, esta actividad tiene una doble cara, es prohibida legalmente, por tanto, los recintos donde se ofrecen estos servicios, son lugares con patentes de bares, cabaret y otras similares que han aumentado significativamente en las últimas décadas en ambas comunas. Tanto en la comuna de Salamanca como de Illapel, existen algunas cuadras reconocidas por la comunidad en donde se concentran estos recintos y existe el comercio sexual, algo así como un barrio rojo. De esta transformación espacial, derivan más bien prejuicios y tensión territorial, no solo por la actividad sino porque se relaciona con delincuencia, drogas, con enfermedades venéreas y también con hombres y mujeres migrantes. Otro foco de prejuicios y tensión en estos territorios.

Si bien, los y las entrevistadas mencionan que existiría una relación entre prostitución, consumo de alcohol, drogas y violencia en territorios mineros, aquello ha sido insuficientemente estudiado (Salinas y Barrientos, 2011). Esto puede relacionarse con la dificultad para acceder a los datos, por ejemplo de violencia doméstica y sexual, sin embargo, las entrevistadas indican que el aumento significativo de bares y lugares nocturnos, en ambas comunas existe un "barrio nocturno" lo ha traído consigo diversos problemas en el radio urbano que incluyen la violencia callejera disminuyendo la calidad de vida.

Se ha expuesto una serie de dimensiones en las dinámicas de género constituidas en territorios mineros, con lo cual se establece que aquellas, profundizan y perpetúan los roles de género tradicionales, con ello las mujeres están doblemente afectadas, por una parte, les es difícil participar de los procesos económicos y políticos de estos territorios, lo que incluye la inserción laboral, debido principalmente a que la minería, impone un modelo hecho para los hombres, lo que, por otra parte, provoca que esta marginación se reproduzca y profundice en otras esferas de la vida social y doméstica. Para ellas, transformar estas dinámicas desde sus limitadas posibilidades es un enorme desafío, como menciona una entrevistada: "estamos peleando con la humanidad misma, no es tan fácil cambiar las mentalidades, la cultura, eso muy difícil, tienen culpa y miedo de ser apedreadas en la plaza" (Comunicación personal Programa jefas de hogar Illapel 14 agosto 2019), y este desafío no debe o puede ser asumido solamente por ellas, sino que debe ser resuelto por la sociedad que produce estas dinámicas y que continua excluyendo a las mujeres, promoviendo con ello, un cambio cultural que permita que los territorios mineros tengan impacto positivo en el desarrollo local y en la vida de los habitantes. 


\section{Reflexión final: dinámicas de género en territorios mineros}

En Chile, existe una cultura minera masculinizada inspirada en una larga tradición en la cual la mujer no ha desempeñado roles importantes. Chile reconoció la presencia de mano de obra femenina recién a mediados de los años 90 , pues antes no estaba permitido que las mujeres trabajen en minería. Este sesgo genera que actualmente tanto las políticas de recursos humanos como las prácticas organizacionales sigan desarrollándose sin considerar las necesidades particulares de sus trabajadoras (Stefanovic \& Saavedra, 2016), imponiendo con ellas una limitación en su inserción laboral y con ello una marginación social.

Esta construcción del género en la minería, se refleja en los territorios producidos por la industria, donde su discurso de desarrollo, crecimiento y empleo instala imaginarios en estos territorios. Sin embargo, las comunidades no solo han cuestionado y resistido a la minería por los daños ambientales sino porque los efectos sobre el desarrollo local no cumplen las expectativas o proyecciones instaladas o comprometidas. De este modo, las dinámicas de género producidas por la minería constituyen una evidencia más de sus impactos y de la situación de marginación que viven las mujeres en estos territorios. Si bien la industria ha mostrado en la última década una perspectiva inclusiva, la "cultura minera" como producto de las formas en que esta industria se relaciona con sus trabajadores y territorios, no permite que sea suficiente para modificar aquellas dinámicas.

El valle del Choapa es un territorio que ha experimentado las transformaciones de la minería desde hace más de 30 años y actualmente sigue teniendo enormes desafíos para su desarrollo. En este sentido la situación de marginación social que viven las mujeres es una evidencia de ello. La construcción de género producto de la minería, ha profundizado las desigualdades entre mujeres y hombres, aumentando las limitantes que ellas deben enfrentar para la inclusión laboral en el territorio pero también mayores desafíos en la vida social y doméstica.

Si bien, la perspectiva de género permite analizar los problemas y las desigualdades que enfrentan las mujeres, así como identificar los factores que reproducen las inequidades, en definitiva, se llega a analizar y develar a los hombres (Lagarde, 1996), puesto que las evidencias aquí encontradas se traducen en como las prácticas masculinizadas de los territorios mineros, son la principal causa y fuente de las marginaciones experimentadas por las mujeres (Caro et al., 2020). Una industria masculinizada como la minería, debe modificarse para incluir a las mujeres, no solo con una mayor oferta de empleos, sino que estos empleos deben estar diseñados para ellas sus condiciones y posibilidades. Por tanto, esta perspectiva lejos de ser sesgada, tiene un contenido relacional. Poniendo en consideración, que los asuntos de género no son un problema de una lista y que no se resolverán a corto plazo ni con cambio de enfoques, se necesitarían un cambio normativo y en enfuerzo tanto en lo público como en lo privado para hacer que la minería tenga impactos positivos en los territorios desde esta perspectiva.

Como se describió las limitantes son variadas, no hay un número de empleos suficientes para ellas por causas diversas, los empleos ofrecidos siguen siendo incompatibles con la vida de las mujeres de estas comunas, adicionalmente, los empleos son de menores jerarquías y salarios, 
tanto dentro de la mina como fuera de ella, situación que no permite que ellas puedan pagar por el cuidado de hijos u otros familiares.

Este análisis sobre la inserción laboral de las mujeres se hace relevante no solo, porque son evidencias que ellas no pueden acceder de forma igualitaria, siendo discriminadas del proceso económico y político que se está produciendo en el territorio en que viven, sino, además, porque dichas desigualdades se traspasan a otras esferas e intersectan con otras dimensiones de la vida social y doméstica. Asimismo, la importancia que tiene para ellas la inclusión laboral en estos territorios, no es necesariamente una muestra de la legitimación de la industria, sino más bien, es producto de la dependencia que ha generado Minera Los Pelambres, dominando el mercado laboral.

Esta mirada inicial y exploratoria de las mujeres en territorios mineros, que intenta avanzar en la integración de espacios domésticos en la investigación territorial, invita a profundizar en las múltiples dimensiones del impacto local de la minería y sobre todo del efecto diferenciado que tiene en las mujeres y en las familias. No sólo para continuar evidenciando que los impactos negativos de la minería en los espacios locales son mayores que los beneficios para estas poblaciones teniendo que diseñar formas de reducir, limitar y compensar dichos daños, sino porque esta desigualdad se profundiza en ciertos grupos de la población que han sido históricamente marginados.

Este trabajo fue desarrollado gracias al financiamiento de la Universidad Academia de Humanismo Cristiano a través de un proyecto FIIC.

\section{Referencias bibliográficas}

ANGELCOS, N. Violencia y táctica en los procesos de integración de las mujeres a la minería del cobre en Chile. Psicoperspectivas. Individuo y Sociedad, 2017, Vol.16, N², p.66-78.

AROCA, P. y ATIENZA, M. La conmutación regional en Chile y su impacto en la Región de Antofagasta. EURE (Santiago), 2008, Vol.34, Nº102, pp.97-120.

AYALA, E. Mujer y Minería: situación de la industria chilena. Tesis para optar al grado de Magíster en Gestión de Personas, Facultad de Economía y Negocio, Universidad de Chile. 2012.

BARRIENTOS, J., SALINAS, P., ROJAS, P. y MEZA, P. Minería, género y cultura. Una aproximación etnográfica a espacios de esparcimiento y diversión masculina en el norte de Chile. AIBR. Revista de Antropología Iberoamericana, 2009, Vol.4, No 3, p.385-408.

BEBBINGTON, A. (2013). Industrias extractivas, conflicto social y dinámicas institucionales en la región andina. Lima: Instituto de Estudios Peruanos

BLANCO, C. y DONGO, M. Género e industrias extractivas en América Latina: medidas estatales frente a impactos diferenciados en las mujeres. Derecho, Ambiente y Recursos Naturales DAR, Lima-Perú. 2019. 
BUSTOS-GALLARDO, B., y PRIETO, M. Nuevas aproximaciones teóricas a las regiones-commodity desde la ecología política. Revista EURE - Revista de Estudios Urbano Regionales, 2019, Vol. 45, No 135, p. 153-176.

CAMPOS, L.; GODOY, R.; TOLEDO, N. y QUINTANA, S. Desde la educación superior abriendo espacios de discusión y reflexión para la participación e inserción igualitaria del género femenino en el sector minero. Círculo de mujeres mineras UDA, 2016.

CARO, P., ILABACA, P., ROMÁN, H., ARMIJO, L., CELIS, K. y MEYER, L. Inclusión sustentable de mujeres en industrias masculinizadas. La minería en Chile. Ediciones Universidad Santo Tomas: Santiago de Chile, 2019.

CARO, P., ROMÁN, H. Y ARMIJO, L. Cuerpos de mujeres, significados de género y límites simbólicos en la gran minería en Chile. Polis. 2020, № 55 2020, p.186-211.

CENSO. Censo de Población y Vivienda Chile, 2017. Disponible en Internet: https://www.censo2017.cl

CODELCO. Historia de CODELCO, 2007. Disponible en Internet: http://www.codelco.com/la_corporacion/historia.asp.

CONSEJO DE COMPETENCIAS MINERAS Mujer y Minería: evolución en la última década y desafíos futuros. Consejo minero y Fundación Chile. 2020a. Disponible en Internet: https://fch.cl/wp-content/uploads/2020/09/mujermineriaccm_02-09-2020.pdf

CONSEJO DE COMPETENCIAS MINERAS Empleo local en la gran minería chilena: una mirada a la actualidad, iniciativas y próximos desafíos, 2020b. Disponible en Internet: https://www.ccm.cl/ wp-content/uploads/2020/12/EmpleoLocalCCM_dic-2020.pdf

CONSEJO DE COMPETENCIAS MINERAS. Fuerza Laboral de La Gran Minería Chilena 2015-2024. Diagnóstico y recomendaciones. Consejo Minero y Innovum Fundación Chile, 2019. Disponible en Internet: https://www.ccm.cl/wp-content/uploads/2016/07/Reporte-28-07-1.pdf

CONSEJO MINERO Mesa Nacional Mujer y Minería 2018-2020. Buenas prácticas de género para el sector, 2020. Disponible en Internet: https://consejominero.cl/wpcontent/uploads/2020/07/Banco_de_buenas_practicas_de_genero-Minmineria_y_Minmujer.pdf

CONSEJO MINERO La mujer en la minería, 2019. Disponible en Internet: https://consejominero.cl/ chile-pais-minero/aprende-de-mineria/mujer-en-la-mineria/

CONTRERAS, D. y PLAZA, G. Cultural factors in women's labor force participation in Chile. Feminist Economics, 2010, Vol.16 No2, p.27-46.

DAHER, A. Regiones-commodities. Crisis y contagio en Chile. Revista EURE, 2003, Vol.29, №86, p.89-108. 
DE LA PUENTE, L. Mujeres y empleo extractivo en América Latina. Politai: Revista de Ciencia Política, Año 8, 2017, No 15. p. 43-62

DÍAZ, E. Mujeres en trabajos de hombres: segregación ocupacional y condiciones laborales en los sectores minería y construcción. Cuadernos de investigación, №49, 2014. Departamento de estudios de la Dirección del Trabajo-Santiago de Chile.

EFTIMIE, A., HELLER, K. y STRONGMAN, J. Gender Dimensions of the Extractive Industries: Mining for Equity. The World Bank. 2009.

ESCALONA, D. Visiones de desarrollo y narrativas de resistencia. Prácticas y discursos del conflicto ambiental en contextos del extractivismo minero. Tesis para la obtención del grado de Doctor en Geografía. Pontificia Universidad Católica de Chile. 2020.

GARCÉS, E. Las ciudades del cobre. Del campamento de montaña al hotel minero como variaciones de la company town. Revista EURE, 2003, Vol. XXIX, Nº8, p.131-148.

GÓMEZ, P. y ANGELCOS, N. Equidad de género en la gran minería del cobre de Chile. Experiencias de inserción laboral femenina en espacios masculinizados. Revista Temas Sociológicos, 2018, No22, 49-85.

GUTIÉRREZ, M. (comp.) Género, Familia y Trabajo: Rupturas y continuidades. Desafíos para la investigación política. CLACSO, Buenos Aires. 2007.

INSTITUTO NACIONAL DE ESTADÍSTICAS-INE. Encuesta Nacional de Empleo -ENE. Trimestre OND 2019.

JENKINS, K. Women, mining and development: An emerging research agenda. The Extractive Industries and Society, 2014, Vol. 1, N², p. 329-339.

JIMÉNEZ, C.; ROJAS, P. y TRONCOSO, R. Género, trabajo y subjetividad: el lugar de la mujer en la minería. Revista Persona y Sociedad, 2014, Vol. XXVIII, No 3, p. 65-95.

LAHIRI-DUTT, K. The Megaproject of Mining: A Feminist Critique. En: S. Brunn ed. Engineering Earth Springer. 2010.

LAHIRI-DUTT, K. y MACINTYRE, M. eds. Women Miners in Developing Countries: Pit Women and Others. Aldershot: Ashgate, 2006

LAGARDE, M. El género: La perspectiva de género. En Género y feminismo. Desarrollo humano y democracia, Ed. horas y HORAS, España, 1996, p. 13-38.

LAMALICE, A. y KLEIN, J.L. Efectos socioterritoriales de la mega minería y reacción social: el caso de Minera Alumbrera en la provincia de Catamarca. Revista de Geografía Norte Grande, 2016, No65, 155-177. 
LEY N²1.122 Código del Trabajo. Diario Oficial de la República de Chile, Santiago, Chile, 28 de noviembre de 2018.

MACDONALD, C. The role of gender in the extractives industries. WIDER Working Paper, $\mathrm{N}^{\circ} 52$, 2017. The United Nations University World Institute for Development Economics Research (UNU-WIDER), Helsinki.

MINERA LOS PELAMBRES Empleabilidad local. 2017. Disponible en Internet: https://web.pelambres.cl/comunicaciones/noticias/2017/empleabilidad-local-un-compromiso-que-une-a-minera-los-pelambres-y-los-municipios-de-la-provincia/

MOGUILLANSKY, G. Chile: las inversiones en el sector minero 1980-2000. Naciones Unidas. Comisión económica para América Latina y el Caribe.1998.

OIT-ORGANIZACIÓN INTERNACIONAL DEL TRABAJO World Employment and Social Outlook - Trends 2020. Disponible en Internet: https://www.ilo.org/global/research/global-reports/ weso/2020/WCMS_734479/lang--en/index.htm

OLAVARRÍA, J. De la Identidad a la Política: Masculinidades y Política Públicas: Auge y Ocaso de la Familia Nuclear Patriarcales en el Siglo XX. En J. Olavarría y R. Parrini (Eds.), Hombres: Identidad/es y Violencia. Encuentro de Estudios de Masculinidades: Identidades, cuerpos, violencia y políticas públicas FLACSO- Chile, Universidad Academia de Humanismo Cristiano/Red de Masculinidades, 2000, p. 11-28

ROMAN, O., RIOS, A. Y TRAVERSO, J. Barreras de género en el desarrollo profesional de mujeres técnicas de la construcción. Revista de la Construcción 87, 2013, Vol. 12, No 1, p.87-99.

SALAZAR, H. y RODRÍGUEZ, M. Miradas en el territorio: Cómo mujeres y hombres enfrentan la minería Aproximaciones a tres comunidades mineras en México. Primera edición: 2015, Heinrich Boll.

SALAZAR, H. El extractivismo desde el enfoque de género: una contribución en las estrategias para la defensa del territorio. Sociedad y ambiente, 2017, N013, p.35-57.

SALINAS, P. Los discursos masculinos como dispositivos de control y tensión en la configuración del liderazgo y empoderamiento femenino". Revista de Estudios Feministas del Centro de Filosofía y Ciencias Humanas, 2007, Vol. 15, № 3, p.541-562.

SALINAS, P., REYES, C., ROMANI, G. Y ZIEDE, M. Mercado laboral femenino. Un estudio empírico, desde la perspectiva de la demanda, en la región minera de Antofagasta, Chile. Innovar, 2010, Vol.20, No38, pp.125-140.

SALINAS, P. y BARRIENTOS, J. Los discursos de las garzonas en las salas de cerveza del norte de Chile: Género y discriminación. Polis (Santiago), 2011, Vol. 10(29), p.433-461.

SALINAS, P.; BARRIENTOS, J. y ROJAS, P. Discursos sobre la discriminación de género en los trabajadores mineros del norte de Chile. Revista Atenea, 2012, 139-158. 
SALINAS, P. y ROMANI, G. Barreras de género en la minería chilena: una gestión estratégica. Academia Revista Latinoamericana de Administración 2014, Vol. 27, № 1, p. 92-107.

SALINAS, P. y ARANCIBIA, S. Discursos Masculinos sobre el Poder de las Mujeres en Chile. Sujetos y Subjetividades. Revista Última Década. CIDPA, 2006, Vol. 14, N²5, p. 65-90.

SISTEMA DE EVALUACIÓN DE IMPACTO AMBIENTAL-SEIA (1997) Estudio Impacto Ambiental: Proyecto Expansión Minera Los Pelambres 85.00 tpd.

SERVICIO NACIONAL DE LA MUJER Agenda regional de género región de Antofagasta 2008-2010. 2009. Disponible en Internet: http://www.sernam.cl

STEFANOVIC, A. y SAAVEDRA, M. Las mujeres en el sector minero de Chile. Propuestas para políticas públicas de igualdad. Santiago: CEPAL. 2016.

SVAMPA, M. y ANTONELLI M. (eds.) Minería transnacional, narrativas de desarrollo y resistencias sociales, Buenos Aires: Editorial Biblios, 2009.

ULLOA, A. Feminismos territoriales en América Latina: defensas de la vida frente a los extractivismos. Nómadas, 2016, N45, pp. 123-139.

VALDÉS, X., REBOLLEDO, L., PAVEZ, J. y HERNÁNDEZ,G. Trabajos y familias en el neoliberalismo. Hombres y mujeres en faenas de la uva, el salmón y el cobre. Santiago: LOM, 2014.

WARD, B. y STRONGMAN, J. Gender-Sensitive Approaches for the Extractive Industry in Peru: Improving the Impact on Women in Poverty and Their Families. The World Bank, 2011.

WAZIERS, B. y MORALES, O. Como integrar el enfoque de género en el sector de infraestructura. Número especial de infraestructura para el desarrollo $N^{\circ} 2,2020$. Departamento de infraestructura y energía del BID. Disponible en Internet: https://publications.iadb.org/publications/spanish/ document/Como-integrar-el-enfoque-de-genero-en-el-sector-de-infraestructura-Numero-especial-de-Infraestructura-para-el-desarrollo.pdf

ZAMBRA, A., Y ARRIAGADA, E. Género y conflictos socioambientales: Una experiencia de investigación-acción participativa con mujeres dirigentes. Revista de Sociología, 34(1), 2019, p.147-165. 